\title{
“From Nail Bed to Nail Head": An Unusual Case of Multiple Penetrating Nails over Head
}

\author{
Sudhansu Sekhar Mishra ${ }^{1}$ Bikash Ranjan Behera ${ }^{1}$ Srikanta Das ${ }^{1}$ Somnath Prasad Jena ${ }^{1}$ \\ Sitansu Kumar Rout ${ }^{1}$ \\ ${ }^{1}$ Department of Neurosurgery, SCB Medical College, Cuttack, \\ Odisha, India

\begin{abstract}
Address for correspondence Dr. Bikash Ranjan Behera, MS Surgery, B/L -29, VSS Nagar, P.O. Sahidnagar, Bhubaneswar, Odisha 751007,
\end{abstract} \\ India (e-mail: drvikasranjanscb@gmail.com).
}

Indian J Neurotrauma 2015;12:71-74.

\author{
Abstract \\ Keywords \\ - penetrating head \\ injury \\ - nails \\ - foreign bodies \\ - craniectomy
}

Multiple penetrating brain injuries are very common in military practice but not so common in civilian road traffic accidents. A rare case of penetrating head injury resulting from a road traffic accident has been presented. The 22-year-old man presented to us after encountering an accident in his maiden motorbike ride. In this accident he lost control of the bike and was catapulted of the bike unto a fence nearby. This fence had been constructed recently and had nails embedded in it. The result of this impact was this young man's skull being impregnated with three nails. With these nails he was retrieved from the site conscious, oriented, and was referred to our neurotrauma facility. On examination he had a GCS of E4V5M6, without any clinical features of raised intracranial pressure (ICP). The local examination suggested three iron nails in the right frontal region, which were firmly fixed to the underlying skull. The CT scan confirmed these clinical findings and an additional finding of a dural penetration and penetration of brain parenchyma. The patient underwent a prompt surgery, with the margin of the skin flap touching all the three nails and a craniectomy performed in a manner to aid the safe extraction of the nails and examination of underlying dura. The bone was discarded and the patient discharged uneventfully with advice of cranioplasty at a later date.
Multiple penetrating brain injuries are very common in military practice but not so common in civilian road traffic accidents. There are various reports of multiple foreign bodies such as pellets, nails, stones due to blasts, or gunshot injuries. ${ }^{1}$ Cases of penetrating nails injuring the skull and brain have been reported earlier. ${ }^{2}$ However, cases of multiple penetrating nails to the skull following a road traffic accident are rare, and here we are presenting one such unusual case of multiple penetrating nails embedded in right frontal part of calvarium with a very unusual and interesting mode of injury.

\section{Case Report}

A 22-year-old man presented to casualty as a case of multiple penetrating nails in the right side of the skull after motorcycle accident. He presented to us 4 hour after the injury. On taking history, he met with an accident on the very first day of his learning to ride a motorcycle. He could not control the motorcycle and crashed onto the boundary wall, which was newly constructed with multiple nails embedded over it. His head stuck to the nails protruding from the wall after collision. As the wall was constructed 1 day before, the nails easily came out of the wall but stuck to the skull bone. Following this accident he had no loss of consciousness, convulsion, vomiting without any history of ear, nasal, or throat (ENT) bleed. On admission, patient was conscious, oriented, and responding to verbal commands. His vitals were stable: GCS, E4V5M6; pupils, B/L normal size and equally reactive to light. No clinical signs of raised intracranial pressure (ICP). No motor or sensory deficit. On local examination, three iron nails were found on the scalp 


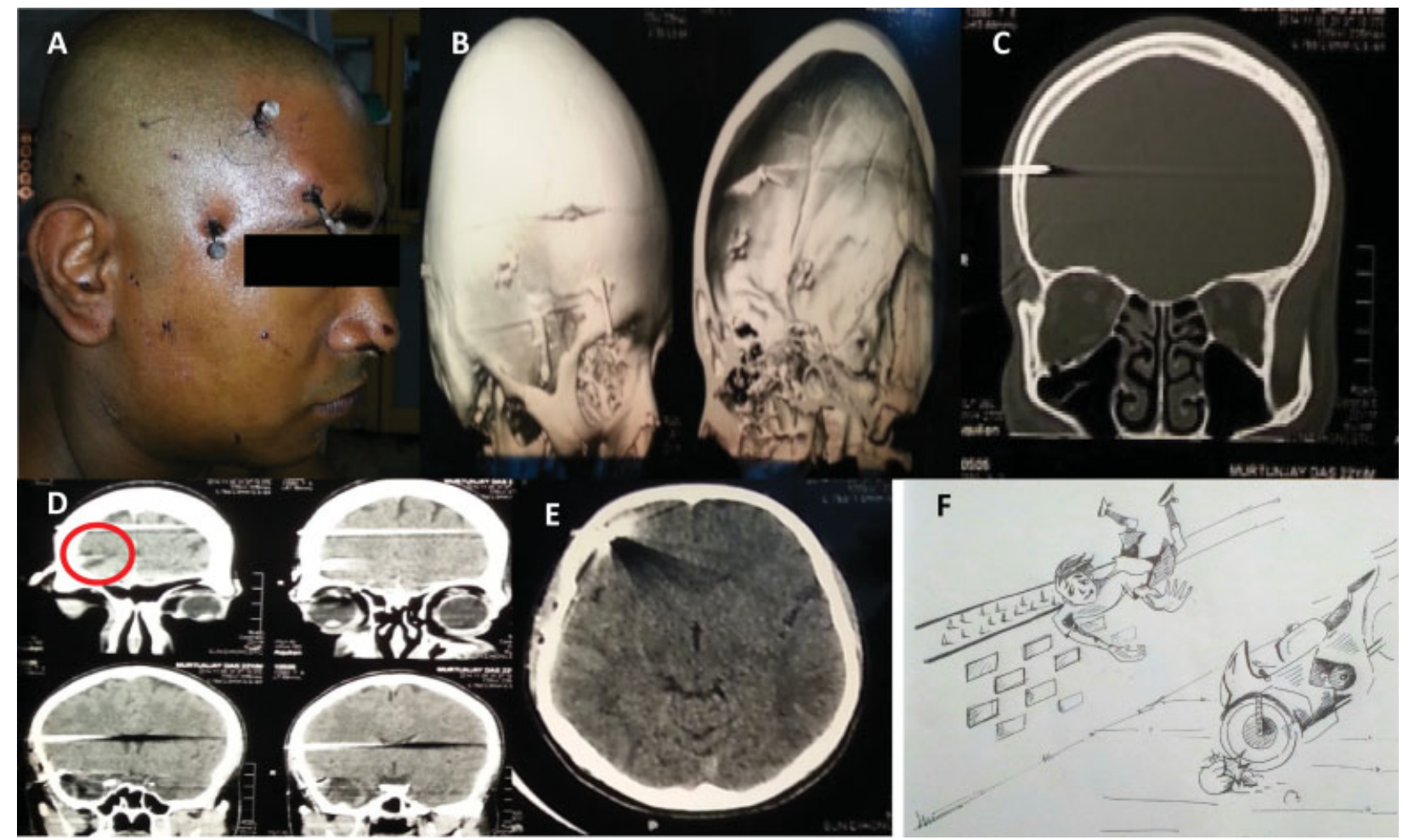

Fig. 1 (A) Clinical photograph of the patient showing the three nails in situ. (B) 3D reconstruction of the skull showing the entry of the nails and the breach of the inner table. (C) Bone window showing the entry of the nail in to the skull. (D, E) NCCT brain showing scattering artifacts in coronal and axial sequence and some evidence of parenchymal injury shown by red ring. (F) Cartoon showing mechanism of injury.

at right frontal region, which were firmly fixed to the underlying bone (although no malicious attempts were made to dislodge them during this local examination). No associated systemic injuries were found including a cervical spine clearance. All laboratory investigations were within normal limit. Noncontrast computed tomography (NCCT) of the brain with 3D reconstruction of the skull bone revealed all three nails penetrated the skull bone with breach of the inner table and also revealed penetration of dura and underlying brain parenchyma in right frontal lobe in front of sylvian fissure (-Fig. 1). No hemorrhagic lesion or associated edema or mass effect was seen. Antibiotics, anticonvulsants, and tetanus immunoglobulin was administered and patient shifted to operation theater. Under general anesthesia, in supine position, lateral frontal horse-shoe-shaped scalp incision was made joining three nails with base toward supraorbital ridge (-Fig. 2). The purpose of fashioning such an incision was to raise a single flap with access to all the three nails and the underlying dura simultaneously, maintaining the viability of the flap. We have joined three nails in horse-shoe manner, keeping the base of the flap toward supraorbital ridge; that is, the skin between the lower two nails was not incised and we have started the skin incision from first lower nail and then progressed to the superior nail and then ended the incision at the second lower nail. As the nails were stuck firmly to the bone, they did not come out on raising the skin flap. As all three nails came in the pathway of incision line, flap could easily be raised without disturbing the nails. Single burr hole was made in between three nails and it was enlarged to a small craniectomy with Kerrison punch, to accommodate all the three nails so that all the nails became loosened and came out easily ( - Fig. 2). Following this, thorough irrigation with dilute hydrogen peroxide and normal saline was done. All nails were removed cautiously to prevent further vascular or parenchymal damage. As the nails were not penetrated deep into parenchyma, and no hematoma or contusion discovered in NCCT, the dura was not opened. The wound was again debrided of any other foreign body with thorough saline irrigation and closed it in layers. Postoperatively the patient recovered well. There was no wound infection or seizure. There was no motor or sensory loss and he was able to communicate verbally in a coherent manner. Post-op scan on fifth day was normal with no hematoma or abscess ( - Fig. 2). Patient was discharged with oral antibiotics and anticonvulsants on seventh day. He is under regular follow-up and there has been no report of fever, headache, or seizure till date.

\section{Discussion}

Multiple penetrating brain injuries are very common in war and military injuries but very rare in civilian head injuries. Penetrating brain injuries can be due to industrial accidents, 

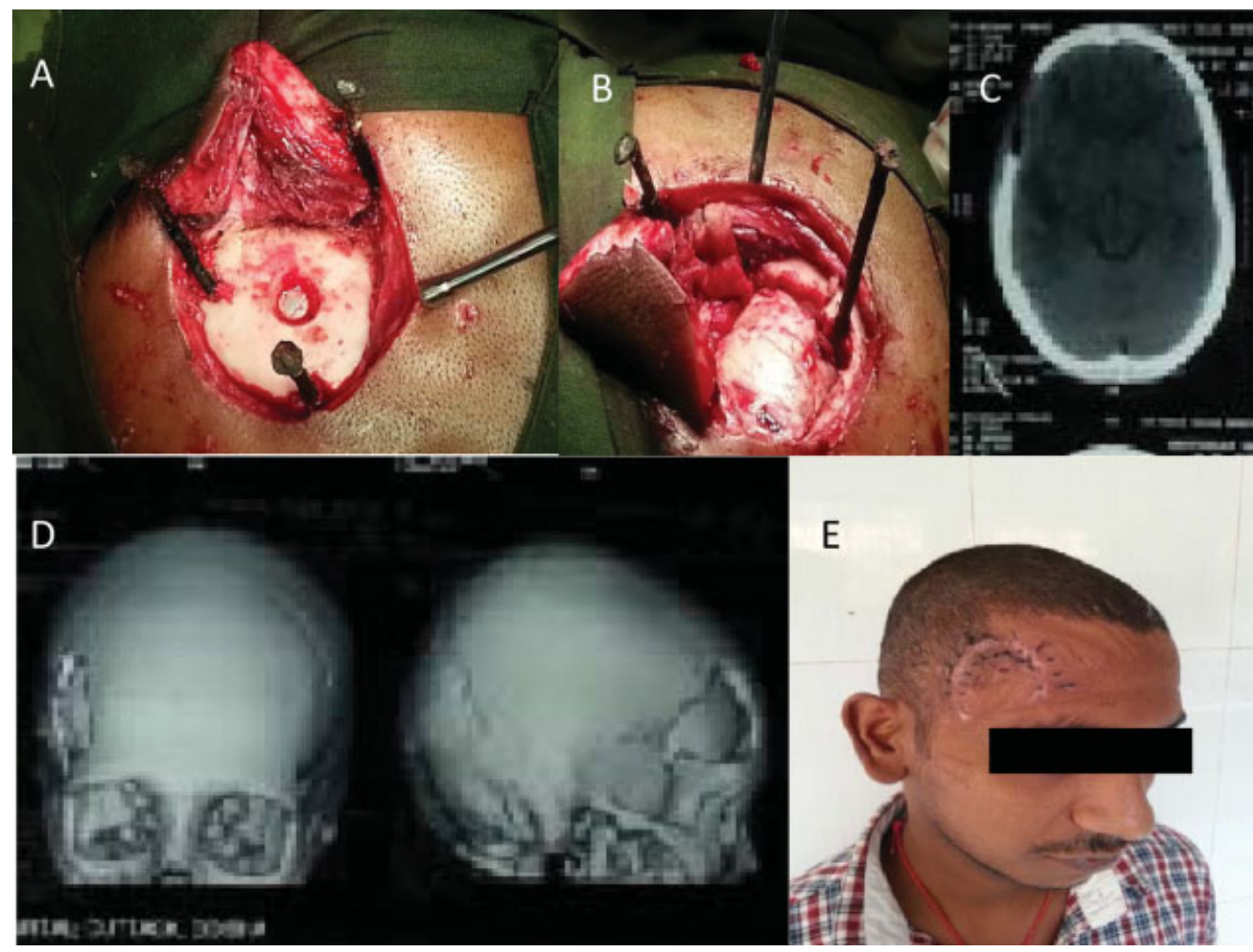

Fig. 2 (A, B) Intraoperative image of curvilinear incision joining the three nails. (C) NCCT brain showing a post-op craniectomy defect in the right frontal region with minimal edema of underlying brain without any mass effect. (D) 3D bone reconstruction showing craniectomy defect. (E) Post-op clinical photograph.

suicide attempts, and criminal assult. ${ }^{1}$ Various objects such as knife, nails, screw drivers, metal rods, and chopsticks are seen in penetrating head injuries. Low-velocity injuries, such as in our case, differ from gunshot and missile injuries in the way that they do not cause concentric cones of cavitations and necrosis. Instead, the damage is predominantly restricted to hemorrhagic infarction and contusion in the line of wound tract. ${ }^{3}$ Low-velocity wounds such as ours are very unlikely to have counter coup or diffuse axonal injury (DAI). Thus, in absence of damage to vital canters and large vessels, the prognosis is usually favorable. Hence, early treatment is necessary in these cases to avoid delayed vascular complications, infections, and epilepsy. Neuroimaging such as NCCT of the brain with coronal, sagittal, axial scans and 3D reconstruction of the skull is essential for decision making regarding surgical intervention. ${ }^{4}$ Cerebral angiography is recommended for patients with penetrating head injuries with increased risk for vascular damage such as wound trajectory passing near the sylvian fissure, supraclinoid carotid, major dural venous sinus, etc. ${ }^{5,6}$ Angiography is also recommended in delayed subarachnoid hemorrhage $(\mathrm{SAH})$ and intracranial hemorrhage (ICH). ${ }^{5,6}$ In our case, no hemorrhage was seen in NCCT and patient had no neurologic deficits; thus, we did not proceed for angiography. Surgical decision varies from center to center. We proceeded with craniectomy rather than circumferential craniotomy for all foreign bodies same time. Generally debridement should be done, but we avoided durotomy as very little part of nail was penetrated into the brain and no hematoma was seen in NCCT. All foreign bodies should be removed under direct vision to avoid further damage to the brain. There is a risk of local wound infection, meningitis, ventriculitis, and cerebral abscess in penetrating brain injuries, with Staphylococcus epidermidis being the most frequently associated organism. ${ }^{7}$ Ideally surgical intervention should be performed within 12 hours of the injury to prevent the risk of infection. ${ }^{8}$ In our case, we operated the case within 10 hours of injury. We followed the recommendations by "The Infection in Neurosurgery Working Party of British Society for Antimicrobial Therapy" and the patient was given inj coamoxiclav (1.2 g) IV 8 hourly with inj metronidazole (500 mg) IV 8 hourly for 5 days. ${ }^{9}$ The risk of posttraumatic epilepsy for penetrating head injuries is approximately 30 to $50 \%$ and it is due to direct traumatic injury to cerebral cortex with subsequent scarring. ${ }^{10}$ Ten percent of patients present with seizure within 7 days of trauma, $80 \%$ during the first 2 years. Recent studies recommend anticonvulsants in first week after penetrating brain injury. ${ }^{10}$ In this case, we have continued prophylactic anticonvulsants and antibiotics for 1 week and there is no seizure or infection till now.

\section{Conclusion}

The rarity of the mechanism of injury and the resulting penetration of multiple nails in the skull make this case an interesting read. A complete radiologic evaluation, careful 
surgical removal of embedded nails by craniectomy, use of antibiotics and antiepileptic drugs, and close postoperative neurologic evaluation may be lifesaving, even in cases of multiple penetrating craniocerebral injuries caused by nails.

\section{Conflicts of Interest}

None.

\section{References}

1 Bakay L, Glasauer FE, Grand W. Unusual intracranial foreign bodies. Report of five cases. Acta Neurochir (Wien) 1977;39(3-4): 219-231

2 Salar G, Costella GB, Mottaran R, Mattana M, Gazzola L, Munari M. Multiple craniocerebral injuries from penetrating nails. Case illustration. J Neurosurg 2004;100(5):963

3 Domingo Z, Peter JC, de Villiers JC. Low-velocity penetrating craniocerebral injury in childhood. Pediatr Neurosurg 1994; 21(1):45-49
4 Offiah C, Twigg S. Imaging assessment of penetrating craniocerebral and spinal trauma. Clin Radiol 2009;64(12): 1146-1157

5 Sani S, Jobe KW, Byrne RW. Successful repair of an intracranial nailgun injury involving the parietal region and the superior sagittal sinus. Case report. J Neurosurg 2005;103(3):567-569

6 Herring CJ, Lumsden AB, Tindall SC. Transcranial stab wounds: a report of three cases and suggestions for management. Neurosurgery 1988;23(5):658-662

7 Hagan RE. Early complications following penetrating wounds of the brain. J Neurosurg 1971;34(2, Pt 1):132-141

8 Benzel EC, Day WT, Kesterson L, et al. Civilian craniocerebral gunshot wounds. Neurosurgery 1991;29(1):67-71, discussion 71-72

9 Bayston R, de Louvois J, Brown EM, Johnston RA, Lees P, Pople IK. Use of antibiotics in penetrating craniocerebral injuries. "Infection in Neurosurgery" Working Party of British Society for Antimicrobial Chemotherapy. Lancet 2000;355(9217): 1813-1817

10 Aarabi B, Taghipour M, Haghnegahdar A, Farokhi M, Mobley L. Prognostic factors in the occurrence of posttraumatic epilepsy after penetrating head injury suffered during military service. Neurosurg Focus 2000;8(1):e1 